ISSN: 2602-8506

Vol. 4, N²., p.5-21 abril - junio, 2020

\title{
La educación financiera y su incidencia en la capacidad de gestión de los comerciantes de la empresa pública municipal Mercado de Productores Agrícolas San Pedro de Riobamba (EP-EMMPA)
}

Financial education and its impact on the management capacity of merchants of the municipal public company Agricultural Producers Market Riobamba's San Pedro (EP-EMMPA)

Victor Oswaldo Cevallos Vique. ${ }^{1}$, Paulina Elizabeth Valverde Aguirre. ${ }^{2}$, Luis Alcides Orna Hidalgo. ${ }^{3} \&$ Edmundo Cargua Pilco. ${ }^{4}$

\begin{abstract}
DOI: https://doi.org/10.33262/visionariodigital.v4i2.1201

The objective of this research was to determine the levels of financial education of the merchants of the public company Agricultural Producers Market Riobamba's San Pedro EPEMMPA, and its impact on management capacity; from a universe of 946 permanent tenant merchants, a representative sample of 195 was obtained, to whom a survey was applied using the random method on aspects related to: level of education and financial knowledge; behavior and attitudes in relation to investment, financing, saving and management of basic financial tools. The relevant findings evidenced the empirical management of finances by the merchants of the Riobamba wholesale market, due to the low level of financial education that affects the ability to manage and manage their economic resources. At the same time,
\end{abstract}

\footnotetext{
${ }^{1}$ Escuela Superior Politécnica de Chimborazo, Facultad de Administración de Empresas, Riobamba, Ecuador, vicevallos@espoch.edu.ec

2 Escuela Superior Politécnica de Chimborazo, Facultad de Ciencias, Riobamba, Ecuador, paulina.valverde@espoch.edu.ec

${ }^{3}$ Escuela Superior Politécnica de Chimborazo, Facultad de Administración de Empresas, Riobamba, Ecuador, luis_orna@espoch.edu.ec

${ }^{4}$ Investigador Independiente, edmuncarg @ hotmail.com
} 
ISSN: 2602-8506

Vol. 4, N²., p.5-21 abril - junio, 2020

they generate serious difficulties such as: over-indebtedness, lack of investment, poor savings and financing of commercial activity through informal sources with high financial and social costs. The research concludes the need for knowledge transfer that allows in the medium and long term to strengthen the financial culture of merchants through financial training and education programs with direct participation of the Universities and Polytechnic Schools of the town through their Management Faculties. Companies and community engagement units

Keywords: Financial education, Management Capacity, Investment, Financing, Savings, Budget.

\section{Resumen}

La presente investigación tuvo como objetivo determinar los niveles de educación financiera de los comerciantes de la empresa pública Mercado de Productores Agrícolas San Pedro de Riobamba EP-EMMPA, y su incidencia en la capacidad de gestión; de un universo de 946 comerciantes arrendatarios permanentes, se obtuvo una muestra representativa de 195 a quienes se aplicó una encuesta mediante el método aleatorio sobre aspectos relacionados con: nivel de formación y conocimientos financieros; comportamiento y actitudes en relación a la inversión, el financiamiento, el ahorro y manejo de herramientas financieras básicas. Los hallazgos relevantes evidenciaron el manejo empírico de las finanzas por parte de los comerciantes del mercado mayorista de la ciudad de Riobamba, debido al bajo nivel de educación financiera que incide en la capacidad de gestión y manejo de sus recursos económicos. Al mismo tiempo generan graves dificultades tales como: sobre endeudamiento, falta de inversión, deficiente ahorro y financiamiento de la actividad comercial a través de fuentes informales con elevados costos financieros y sociales. La investigación concluye la necesidad de trasferencia de conocimientos que permita en el mediano y largo plazo fortalecer la cultura financiera de los comerciantes mediante programa de formación y educación financiera con participación directa de las Universidades y Escuelas Politécnicas de la localidad a través de sus Facultades de Administración de Empresas y unidades de vinculación con la comunidad 
Palabras claves: Educación financiera, Capacidad de Gestión, Inversión, Financiamiento, Ahorro, Presupuesto

\section{Introducción}

La EP_EMMPA, es una empresa pública que tiene como misión la prestación de servicios de mercadeo y comercialización de productos agrícolas, cumpliendo estándares de calidad y eficiencia. La empresa cuenta con 946 comerciantes registrados como arrendatarios permanentes, 50 introductores, 343 arrendatarios temporales y de servicios complementarios, con una movilidad humana de aproximadamente 65.000 personas por mes.” (EP-EMMPA, 2019)

La actividad de los comerciantes por su naturaleza, tienen relación directa con el manejo de las finanzas. Los negocios giran en torno a tres tipos de decisiones financieras básicas: Decisiones de inversión, decisiones de financiamiento y decisiones de operación (Gitman, 2012). En el nivel personal y familiar las finanzas tienen que ver con las decisiones que toman los individuos sobre cómo gastar sus ingresos, que proporción de los mismos ahorrar y cómo invertir sus ahorros.

Sin embargo, la actividad comercial de este importante segmento poblacional de la provincia de Chimborazo, afronta grandes problemas relacionados con la escasa cultura financiera de los comerciantes que influyen directamente en el desarrollo de sus negocios. Factores tales como: bajos niveles de educación y formación académica, falta de capacitación y asistencia técnica, carencia de programas de educación financiera, baja participación de autoridades, directivos y demás actores sociales implicados, entre otros aspectos; dan como resultado prácticas financieras erróneas como sobreendeudamiento, escaso ahorro e inversión, ausencia de planificación, etc.; con efectos sociales que implican inestabilidad emocional de sus propietarios, constantes preocupaciones en el manejo del dinero y que en general afectan la sostenibilidad de sus negocios. La falta de acierto del sistema financiero en cuanto a inclusión de segmentos importantes de la población especialmente de clase económica media y baja, hace que los comerciantes se vean en la necesidad de acudir al uso de fuentes de financiamiento alternativos e informales que generan una cadena de costos económicos y sociales elevados. En el caso de los comerciantes del mercado mayorista San Pedro de Riobamba, es común el endeudamiento a través del chulco, práctica ilegal e inmoral que en muchos casos ha determinado la quiebra de pequeños negocios y familias, afectando la tranquilidad y el bienestar de la comunidad en general. 
ISSN: 2602-8506

Vol. 4, No2., p.5-21 abril - junio, 2020

El éxito o el fracaso de los pequeños negocios y emprendimientos desarrollados por los comerciantes de productos agrícolas de la ciudad de Riobamba dependen en gran medida de la capacidad de gestión de sus propietarios. Por ende, esta investigación se propone responder a la pregunta: ¿De qué manera incide la educación financiera de los comerciantes de la EP-EMMPA, en el desarrollo y mejoramiento de su capacidad de gestión?

Los comerciantes del mercado mayorista San Pedro de Riobamba, son emprendedores que han encontrado en la actividad comercial su fuente de vida, en la generalidad combinan sus labores operativas, administrativas y financieras con la actividad familiar; por ende, requieren herramientas, conocimientos, apoyo educativo para lograr un eficiente desempeño, crecimiento y bienestar colectivo.

\section{Educación Financiera}

La Organización para la Cooperación y el Desarrollo Económico (OECD), define a la educación financiera como el proceso por el cual los consumidores/inversionistas financieros mejoran su comprensión de los productos financieros, los conceptos y los riesgos y a través de información, instrucción y/o el asesoramiento objetivo, desarrollan las habilidades y confianza para ser más conscientes de los riesgos y oportunidades financieras, tomar decisiones informadas, saber a dónde ir para obtener ayuda y ejercer cualquier acción eficaz para mejorar su bienestar económico (OECD - CAF, 2013, citado en Chalacán,2020).

La definición anterior puede ser entendida en un sentido amplio como la necesidad de los individuos para en primer lugar comprender conceptos y nociones básicas de las finanzas, para luego hacer uso a través de la inclusión financiera de productos y servicios que ofertan las entidades públicas y privadas a fin de mejorar su condición de vida.

La educación financiera es un proceso de desarrollo de habilidades y actitudes que, mediante la asimilación de información comprensible y herramientas básicas de administración de recursos y planeación, permite a los individuos: a) tomar decisiones personales y sociales de carácter 
ISSN: 2602-8506

Vol. 4, N²., p.5-21 abril - junio, 2020

económico en su vida cotidiana, y b) utilizar productos y servicios financieros para mejorar su calidad de vida bajo condiciones de certeza (Bansefi, 2008, citado en Cargua 2019).

La educación financiera según el autor Pellas (2008), es un proceso de adquisición de conocimientos, actitudes, destrezas, valores, hábitos y costumbres en el manejo de las finanzas personales para lograr un adecuado manejo de instrumentos financieros básicos. En las definiciones anteriores, se identifica la importancia de la educación financiera en la formación integral de las personas, es decir no solamente en el conocimiento y manejo de herramientas financieras, sino en la parte axiológica de las personas, las familias y la sociedad en general.

Las características de la educación financiera se resumen en los siguientes elementos: Transmite conocimientos, desarrolla habilidades y eleva el nivel de bienestar personal, familiar y el de la sociedad en general. La educación financiera cobra importancia en épocas de inestabilidad e incertidumbre como la que vivimos, la crisis económica y financiera desatada por el coronavirus que apenas empieza, impacta directamente en los niveles de empleo, merma la actividad productiva, comercial y económica de los estados, las familias y personas y pone de manifiesto la necesidad de una cultura del ahorro, de la prevención y previsión de recursos, de la optimización y solidaridad colectiva.

La educación financiera por tanto debería ser parte de la formación de todo tipo de personas y ser incluido como eje trasversal en los pensum de estudios del sistema educativo desde el nivel de educación básica, bachillerato y superior. Mientras más temprano los niños aprendan a valorar la importancia del manejo del dinero, la optimización de recursos, y la generación de oportunidades, existirán mayores posibilidades de mejorar la calidad de vida de la población.

\section{Las finanzas personales}

Las finanzas personales consisten en la aplicación de los principios financieros en la administración de los recursos de una persona o una familia. En su análisis se incluyen no solo los ingresos y gastos recibidos o pagados durante la vida, sino que también las herramientas o productos financieros con los que cuentan los individuos o familias para optimizar sus recursos (Roldan, 2019). 
ISSN: 2602-8506

Vol. 4, N²., p.5-21 abril - junio, 2020

La eficiente gestión de las finanzas personales, tienen que apoyarse en los principios financieros básicos tales como: el ahorro, la inversión, adecuados niveles de endeudamiento, el valor del dinero en el tiempo, no gastar más de lo que ingresa, reinvertir utilidades para generar crecimiento, elaborar presupuestos de ingresos y gastos, separar las cuentas del negocio y de las personas, poner a trabajar al dinero cuando existan excedentes, entre otros (CCLOAN, 2020).

Uno de los problemas que afrontan los comerciantes de la EP: EMMPA precisamente es la falta de separación de las cuentas del negocio con las cuentas familiares, en estos negocios familiares participan varios miembros del núcleo familiar por tanto no se reconocen gastos, como tampoco se reconocen todos los ingresos que permitan identificar y trasparentar los resultados de la gestión comercial.

Las finanzas personales procuran mantener un equilibrio adecuado entre el nivel de ingresos y gastos. Por una parte, será necesario maximizar las fuentes de ingresos familiares diversificándolos y buscando siempre la independencia financiera a fin de no depender exclusivamente de una actividad como por ejemplo de un empleo. Por otra parte; se procurará la mejor distribución del dinero y la optimización de los gastos, considerando en primer lugar las necesidades básicas: alimentación, educación, salud, vivienda, vestimenta, transporte, recreación, etc.

El éxito de las finanzas personales se verá reflejado en el grado de cumplimiento de los objetivos y metas de las personas en el mediano y largo plazo, en el logro y ejecución de su proyecto de vida. El eficiente manejo de las finanzas personales depende básicamente del comportamiento de las personas más que de sus conocimientos; al respecto el autor Dave Ramsey (2019), manifiesta: que el $80 \%$ de las finanzas personales está relacionado con el comportamiento de las personas frente al dinero y el $20 \%$ proviene del conocimiento, debido a que es más factible que las personas estén más interesadas en modificar sus hábitos y costumbres que sus conceptos.

Un principio financiero básico a considerar es: "Invertir en tu educación tanto para mejorar tus negocios como para aprovechar sus frutos" (América Economía, 2015), esto significa que la mejor inversión es la educación sin importar el nivel académico ni social, mediante la educación 
ISSN: 2602-8506

Vol. 4, $\mathrm{N}^{\circ}$ 2., p.5-21 abril - junio, 2020

financiera hay que buscar las herramientas para mejorar la capacidad de gestión y desarrollo de habilidades en los negocios.

\section{El presupuesto}

El presupuesto, es una herramienta de planeación que sirve como guía en todo tipo de entidad, organización, empresa y familia. Su punto de partida es un diagnóstico situacional y el planteamiento de objetivos, estrategias y políticas que se traducen en valores cuantificables, generalmente se los realiza para períodos mensuales, trimestrales o anuales en función de los requerimientos particulares.

El presupuesto es utilizado en los procesos de planeación, ejecución y control administrativo; es una expresión cuantitativa formal de los objetivos que se propone alcanzar la administración de la empresa en un periodo, con la adopción de las estrategias necesarias para lograrlos. (Carbajal, 2013, citado por Cargua, 2019).

Mediante el presupuesto se pueden hacer frente a las incertidumbres que están presentes en todos los aspectos de la vida económica, social, política y administrativa de todo tipo de entidades públicas y privadas, así como también en el desarrollo de la vida de las familias y personas. Ajustarse a un presupuesto significa disciplinarse y no gastar más de lo que se tiene.

Si la planeación ayuda a definir metas y objetivos, la elaboración de un presupuesto permite establecer prioridades, auto disciplinarse en función del conocimiento de cuáles son los ingresos y cuáles son los gastos en un periodo de tiempo determinado; si se conoce estas variables básicas también se podrá definir cuanto destinar al ahorro, la inversión y se podrá igualmente controlar la calidad del gasto.

Al respecto el autor Méndez, G. (2015), manifiesta: "la seguridad financiera de los individuos radica principalmente en la capacidad de poder mantener un nivel de vida apropiado, ahorrar para imprevistos, e invertir para alcanzar objetivos a mediano y largo plazo."

\section{La capacidad de gestión}

En los pequeños negocios como es el caso de los comerciantes de la EP_EMMPA, el papel del emprendedor es el de asumir varias funciones y responsabilidades, en él se concentran 
ISSN: 2602-8506

Vol. 4, $\mathrm{N}^{\circ}$ 2., p.5-21 abril - junio, 2020

prácticamente todas las actividades administrativas, financieras y operativas; $\mathrm{y}$ todas estas actividades tiene que desarrollarlas con altos niveles de eficiencia. Lo descrito anteriormente podría definirse como capacidad de gestión, y de la misma depende la supervivencia, crecimiento y sostenibilidad del negocio. La capacidad de dirigir, de gestionar es el motor impulsor para el desarrollo de un país, de una empresa o de una economía familiar (WIKI EOI, 2013). La gestión supone tres aspectos: conocimientos, voluntad y disposición de recursos; esto significa aptitud, actitud y poder; por tanto, la gestión está relacionada con la esencia misma de la administración como es la toma de decisiones.

Según el autor Juan Carlos Ayala (2005), en su publicación Manual de Competencias básicas en Gestión, manifiesta que la gestión está relacionada con la capacidad de manejar todo tipo de recursos, manifiesta "es la capacidad general que desarrollan las personas para identificar problemas, reconocer información relevante, tomar las decisiones adecuadas, coordinar los recursos disponibles y proveerse de aquellos que no tiene”. En este sentido podemos concluir: que el proceso que trasforma la realidad, que la toca, que la convierte en otra cosa, es la gestión. La gestión comercial por su parte proporciona las técnicas de como promover un producto o servicio, ofrece todas las herramientas necesarias para administrar y gestionar adecuadamente las principales actividades comerciales en mercados cada vez más saturados y competitivos (Miranda, 2018).

\section{Metodología}

\section{Enfoque}

Esta investigación tiene un enfoque predominantemente cualitativo, por cuanto describe una realidad única y busca la comprensión de un fenómeno social como es la educación financiera.

\section{Métodos de investigación}

Se utilizó la investigación de campo, que es aquella que se aplica extrayendo datos e información directamente de la realidad u objeto de estudio mediante el uso de recolección de técnicas como la encuesta y observación para dar respuestas al problema planteado.

La investigación documental bibliográfica, se lo realizó mediante consulta bibliográfica e internet sobre temas educación financiera, así como informes de rendición de cuentas de la empresa y artículos científicos. 
ISSN: 2602-8506

Vol. 4, N²., p.5-21 abril - junio, 2020

\section{Nivel de investigación}

Es una investigación descriptiva, que tiene como objetivo describir y delimitar los diferentes elementos del problema, sin alterar las variables de estudio.

\section{Técnicas e instrumentos de investigación}

Las técnicas que se utilizaron para la recopilación de datos fueron la encuesta y la observación; y los instrumentos utilizados fueron el cuestionario y la guía de observación.

\section{Población y Muestra}

La población de estudio está constituida por 946 comerciantes, que actualmente se encuentran inscritos en calidad de arrendatarios permanentes de la EP_EMMPA, los cuales desarrollan su actividad comercial en forma habitual. Mediante fórmula empleada se obtuvo una muestra de 195 comerciantes a quienes se aplicó la encuesta.

La fórmula aplicada corresponde a poblaciones finitas (Herrera, 2011).

$$
\mathrm{n}=\frac{Z^{2} * p * q}{e^{2}(N-1)+Z^{2} * p * q}
$$

Donde:

- $\quad \mathrm{N}=$ tamaño de la población

- $\quad \mathrm{Z}=1,96$ al cuadrado (si la seguridad es del $95 \%$ )

- $\quad \mathrm{p}=$ proporción esperada (en este caso $5 \%=0.05)$

- $\quad \mathrm{q}=1-\mathrm{p}$ (en este caso $1-0.05=0.95)$

- $\quad$ e= precisión (se utilizó el 5\%)

A continuación, se describe el proceso que se utilizó para el proceso de investigación:

1. Definición y planteamiento del problema

2. Formulación de objetivos

3. Selección de métodos y técnicas de investigación

4. Recopilación de información bibliográfica y documental

5. Obtención de la muestra

6. Elaboración del cuestionario

7. Modificaciones y elaboración cuestionario final previa encuesta piloto. 
ISSN: 2602-8506

Vol. 4, N²., p.5-21 abril - junio, 2020

8. Procesamiento y análisis de datos

9. Revisión de resultados y obtención de conclusiones.

\section{Resultados}

\section{Perfil del grupo de estudio:}

Según información recopilada mediante la encuesta los comerciantes del Mercado Mayorista San Pedro de Riobamba tienen las siguientes características demográficas:

El 65\% de comerciantes son mujeres, el $41 \%$ se encuentra en un rango de edad entre 30 y 40 años, y el 25\% entre los 40 y 50 años. El 37\% tiene un nivel de formación secundaria, el 29\% formación secundaria, $18 \%$ tiene una formación universitaria y el $15 \%$ restante manifiesta no tener ninguna instrucción académica. Es decir que los comerciantes mayoritariamente apenas han culminado la educación primaria y secundaria. Con el propósito de evidenciar el grado de educación financiera de los comerciantes del Mercado Mayorista San Pedro de Riobamba, se procedió a elaborar preguntas relacionadas con tres aspectos: conocimientos, comportamiento y actitud en aspectos financieros básicos. Los resultados obtenidos luego de aplicar las encuestas, fueron los siguientes:

\section{Conocimientos:}

Pregunta 1. ¿Considera que su grado de conocimiento financiero para ejercer la actividad comercial es?:

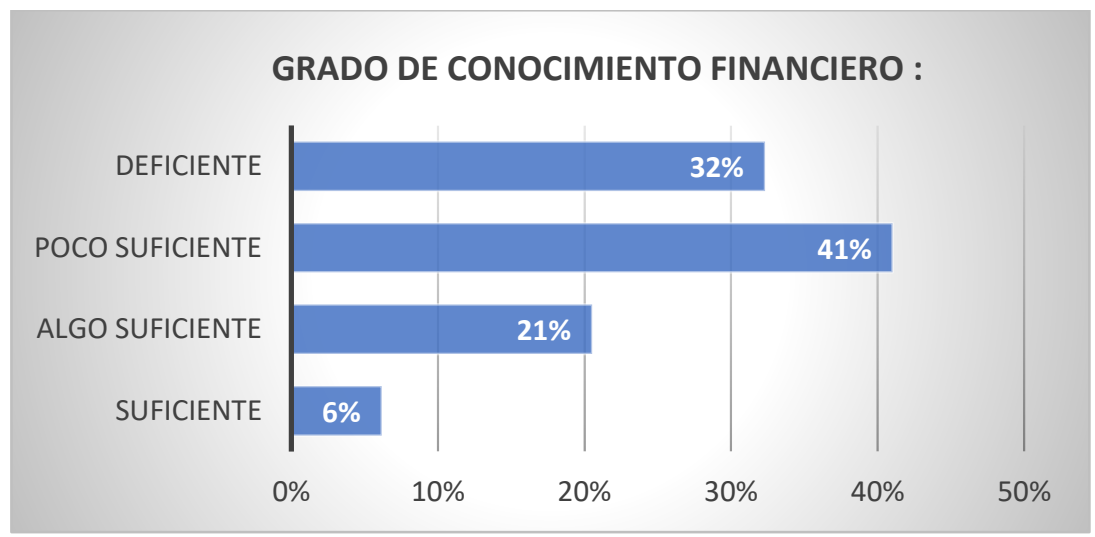

Figura 1. Grado de conocimiento financiero para ejercer la actividad comercial

Elaborado por: Grupo de Investigación.

\section{Análisis de la pregunta 1}

Se pudo establecer que el $41 \%$ de los comerciantes consideran que su grado de conocimiento financiero para ejercer su actividad comercial es poco suficiente; el $32 \%$ considera que es 
ISSN: 2602-8506

deficiente, el $21 \%$ Algo suficiente y solo el $6 \%$ considera suficiente. Es decir que mayoritariamente los comerciantes de la EP_EMMPA, reconocen tener un bajo nivel de conocimiento financiero.

\section{Pregunta 2. ¿De quién aprendió el manejo de las finanzas?}

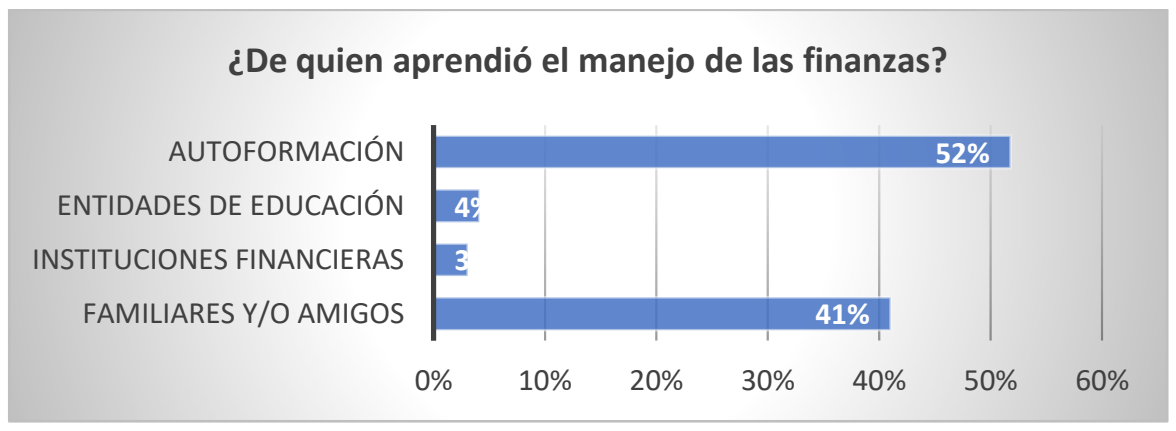

Figura 2 ¿De quién aprendió el manejo de las finanzas?

Elaborado por: Grupo de Investigación

\section{Análisis de la Pregunta 2}

En cuanto a cómo y con quien aprendió el manejo de las finanzas, los encuestados respondieron el $52 \%$ a través de Autoformación, seguido de $41 \%$ mediante influencia de familiares y amigos, en tanto que el $4 \%$ mediante entidades de educación, y apenas el 3\% a través de entidades financieras. Esto significa la escasa o nula participación de entidades de educación superior e instituciones financieras en programas de educación financiera de la población.

\section{Comportamiento y Actitud:}

Pregunta 3. ¿Se pone usted metas económicas a largo plazo y se esfuerza por conseguirlas?

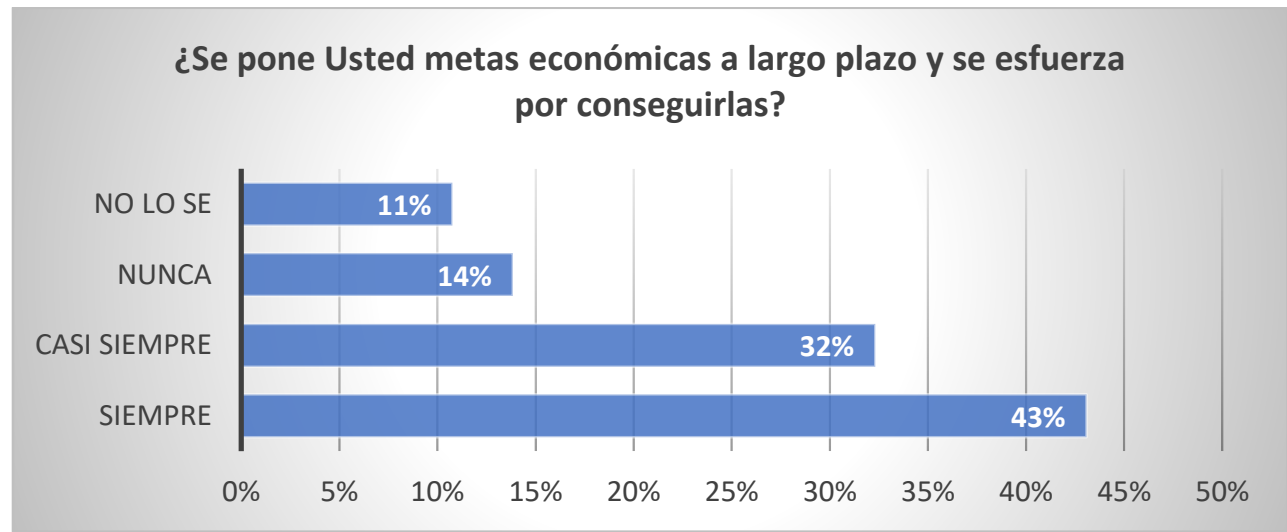

Figura 3 ¿Se pone usted metas económicas a largo plazo y se esfuerza por conseguirlas? Elaborado por: Grupo de Investigación 
ISSN: 2602-8506

Vol. 4, N²., p.5-21 abril - junio, 2020

\section{Análisis de la Pregunta 3}

Una vez tabulada esta pregunta encontramos que el $43 \%$ de encuestados manifiesta que Siempre se pone metas económicas a largo plazo y se esfuerza por conseguirlas, el 32\% Casi siempre lo hace; el $14 \%$ Nunca y el $11 \%$ no sabe. Esa situación refleja la actitud de los encuestados ante la

Inversión; cuando hablamos de metas a largo plazo nos referimos por ejemplo a invertir recursos que le permitan ampliar su negocio, comprar un local o adquirir un medio de transporte para los productos que comercializa, etc.

Pregunta 4. ¿Prefiere usted gastar el dinero hoy, que ahorrar para el futuro?

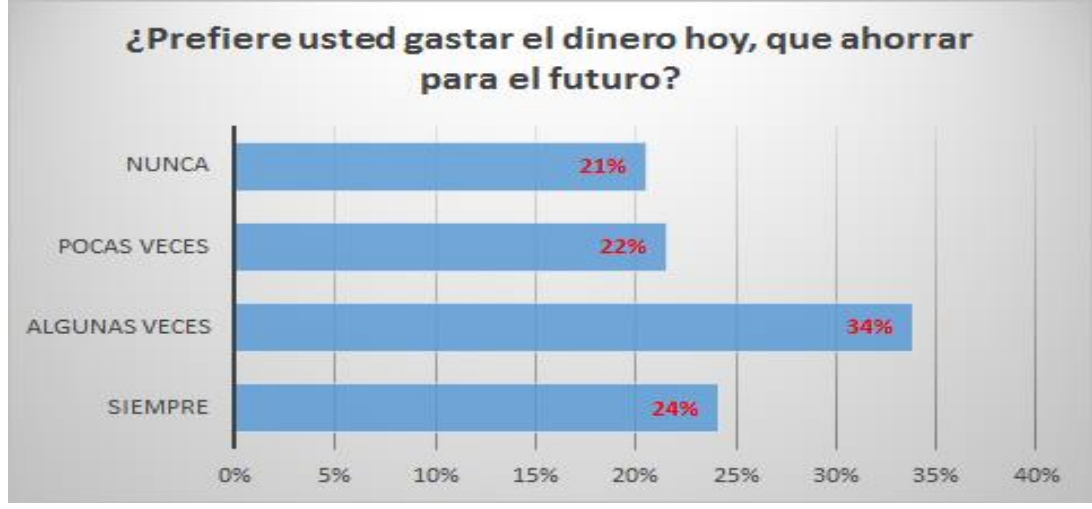

Figura 4. ¿Prefiere usted gastar el dinero hoy, que ahorrar para el futuro?

Elaborado por: Grupo de Investigación

\section{Análisis de la Pregunta 4}

En la pregunta 4 podemos apreciar que el 34\% de los Comerciantes de la EP-EMMPA, algunas veces prefieren gastar el dinero hoy, que ahorrar para el futuro; el $24 \%$ siempre, el $22 \%$ pocas veces y $21 \%$. Esta situación refleja la actitud que tienen las personas frente al Ahorro; en este caso particular más de la mitad de encuestados, no tiene el hábito del ahorro que le permita cubrir en el futuro posibles contingencias o necesidades.

Las personas prefieren gozar de los beneficios presentes, renunciando a un posible bienestar en el mañana. 
Pregunta 5. ¿Cuánto de sus ingresos destina al ahorro, de manera mensual?

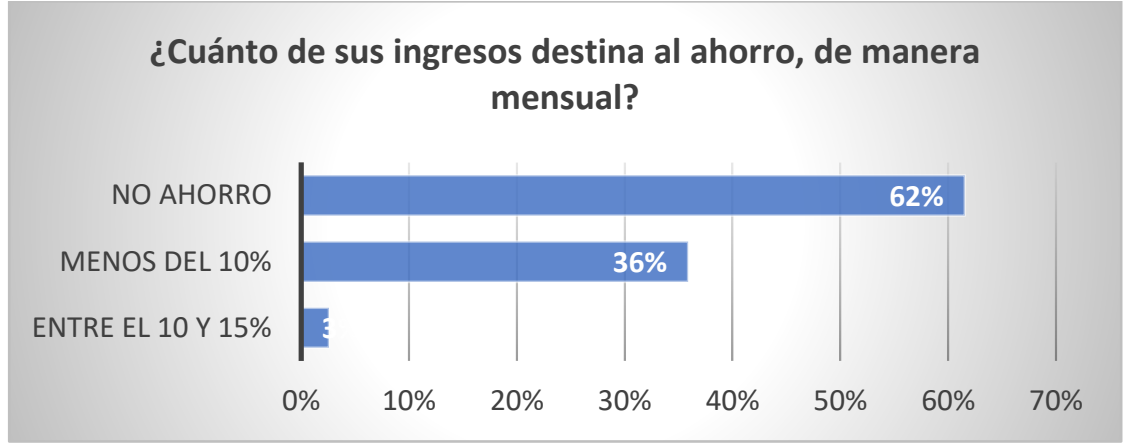

Figura 5 ¿Cuánto de sus ingresos destina al ahorro, de manera mensual?

Elaborado por: Grupo de Investigación

\section{Análisis de la Pregunta 5}

En relación a la Pregunta 5, encontramos que el 62\% de los comerciantes de la EM-EMMPA expresan que no ahorran; el 36\% ahorra menos del 10\% de sus ingresos, y el 3\%, declara que ahorra entre el 10 y $15 \%$ de sus ingresos. Claramente se puede establecer que los comerciantes no tienen un hábito de ahorro, posiblemente está relacionado con la situación económica que actualmente vive el país, caracterizada por la escasez de recursos económicos que apenas permite cubrir necesidades básicas de la población tales como salud, educación, vivienda; por ende no cuenta con la capacidad de destinar recursos al ahorro.

\section{Discusión}

El nivel de conocimientos de aspectos básicos financieros del grupo objeto de estudio es bajo y está directamente relacionado con el bajo nivel de instrucción académica de los comerciantes. Esto significa que a mayor nivel de formación académica hay mayor posibilidad de acceso a la educación financiera. También se establece que los comerciantes tienen un conocimiento empírico producto de su ejercicio diario en la actividad comercial como conocimiento transmitido por amigos y familiares. El planteamiento de metas y objetivos a largo plazo por parte de los comerciantes, no necesariamente significa una actitud orientada a la inversión, en este tipo de negocios informales, no se diferencian los recursos del negocio con los recursos de la familia lo cual se evidencia en el inadecuado manejo del dinero, la falta de registros de ingresos y gastos que impiden en la práctica establecer la utilidad o perdida, menos aún la rentabilidad del negocio. 
ISSN: 2602-8506

Vol. 4, N²., p.5-21 abril - junio, 2020

Las malas prácticas financieras se reflejan en aspectos como altos niveles de endeudamiento, poca cultura de ahorro y escaso interés por la inversión y crecimiento del negocio. Apenas se trabaja para mantener el negocio, dicho de otra manera, para la subsistencia y el día a día. El 51\% de comerciantes de la EP-EMMPA consideran que el porcentaje de ingresos que representan sus deudas es más del 30\%, el 37\% estima que las deudas representan menos del $30 \%$ de sus ingresos y el $11 \%$ desconoce. Se puede deducir altos niveles de endeudamiento que fundamentalmente se orientan a financiar capital de trabajo de los comerciantes en el giro de sus negocios.

Las entidades o personas que han contribuido con financiamiento para el desarrollo de sus actividades comerciales son: en un 49\% las Cooperativas de Ahorro y Crédito, el 22\% mediante préstamos a familiares y/o amigos; el 21\% a través del sistema bancario; y, el 7\% de encuetados también han acudido a buscar financiamiento a través de Otros. Esto significa que ante la necesidad de crédito la primera opción es el sistema cooperativo, no obstante, en el comercio también operan formas ilegales de financiamiento como los denominados Chulqueros que ofertan préstamos a altas tasas de interés ocasionando varios problemas sociales y económicos. Al momento de realizar un crédito por ejemplo no se compara las condiciones entre varias alternativas u otros productos y opciones financieras, apenas un 33\% de encuestados manifestó que si lo hizo. Podemos deducir que mayoritariamente los comerciantes de la EP-EMMPA al momento de endeudarse no se detienen en analizar las condiciones de crédito tales como tasas de interés, métodos de amortización de créditos, garantías, montos, plazos, entre otros.

Al no existir una cultura de ahorro, tampoco existe la posibilidad de cubrirse ante posibles contingencias, por tanto, la práctica común de los comerciantes es recurrir al endeudamiento en aquellas opciones financieras más fáciles, pero a su vez más riesgosas.

El desconocimiento de herramientas financieras básicas hace que los comerciantes del mercado mayorista San Pedro de Riobamba consideren innecesarios el llevar registros contables, elaborar presupuestos, y generar información mínima por ende la toma de decisiones es totalmente empírica. 


\section{Conclusiones}

- La educación financiera tiene una gran repercusión e incidencia sobre la capacidad de gestión en todo tipo de negocios y particularmente de los comerciantes del Mercado Mayorista San Pedro de Riobamba. El escaso conocimiento de las finanzas se refleja en la escasa planificación, organización, dirección y control de los negocios, haciendo ineficiente el uso de recursos y la consecución de objeticos.

- En cuanto al comportamiento y actitud de los comerciantes sobre las variables financieras básicas: Inversión. - aproximadamente el 75\% de comerciantes de la EP-EMMPA tienen definidas sus metas económicas no obstante no se traducen en inversión productiva orientada al crecimiento de sus negocios, esta situación no garantiza la sostenibilidad de sus negocios. Financiamiento. - los comerciantes de EP-EMMPA tiene como principal fuente de financiamiento al sistema cooperativo de la ciudad de Riobamba que cubren sus necesidades de crédito en un 50\%. La otra mitad acuden a familiares y/o amigos y fuentes informales e ilegales denominados chulqueros que se aprovechan de la necesidad de dinero, e imponen altos costos de interés.

- Los comerciantes del mercado Mayorista de la ciudad de Riobamba, no tienen el hábito del Ahorro, aproximadamente un 55\% prefiere gastar el dinero hoy, que ahorrar para hacer frente a posibles contingencias o necesidades futuras. También este indicador está relacionado con la situación económica actual del país que determina una escasa capacidad de ahorro de la población en general.

- Se deduce altos niveles de endeudamiento de los comerciantes a fin de apalancar el giro de sus negocios El 51\% de comerciantes de la EP-EMMPA consideran que el porcentaje sus deudas es mayor al $30 \%$ de sus ingresos.

- Es evidente la escasa participación y aporte de las entidades de Educación Superior y del Sistema Financiero en general en el fortalecimiento de la educación financiera, por lo tanto, es necesario la urgente intervención educativa en programas de educación financiera mediante programas de vinculación con la sociedad. 


\section{Referencias bibliográficas}

América Economía. (2015). Siete tips financieros para emprendedores. Obtenido de https://mba.americaeconomia.com/articulos/notas/siete-tips-financieros-paraemprendedores

Ayala, J. (2005). Manual de competencias básicas en gestión. Obtenido de https://www.oitcinterfor.org/sites/default/files/manual_gestion.pdf

Bansefi. (2008). Obtenido de Definiciones de Educación Financiera: https://www.gob.mx/cms/uploads/attachment/file/83054/Educaci_n_Financiera.pdf

CCLOAN. (2020). La educación financiera y sus principios. Obtenido de https://www.ccloan.es/educacion-financiera

Dave, R. (2019). Consejo Financiero. Obtenido de La transformación total de su dinero: https://consejofinanciero.com/la-transformacion/

EP-EMMPA. (2019). Rendición de Cuentas. Obtenido de http://www.epemmpa.gob.ec/index.php/pages/about-us\#

Gitman, L. J. (2012). Principios de Administración Financiera. México: Pearson Educación.

Herrera, M. (2011). Fórmula para el cálculo de poblaciones finitas. Obtenido de https://investigacionpediahr.files.wordpress.com/2011/01/formula-para-cc3a1lculo-de-lamuestra-poblaciones-finitas-var-categorica.pdf

Méndez, R. G. (2015). La importancia de un presupuesto personal y familiar. Obtenido de http://www.educateyprospera.com/la-importancia-de-un-presupuesto-personal-o-familiar/

Miranda, J. (2018). La gestión comercial y la venta. Obtenido de https://www.gestiopolis.com/lagestion-comercial-la-venta/

OECD - CAF. (2013). Google. Obtenido de La Educación Financiera en América Latina y el Caribe, situación actual y perspectivas: https://www.oecd.org/daf/fin/financialeducation/OECD_CAF_Financial_Education_Latin_AmericaES.pdf

Pellas, C. E. (2008). Libro Maestro de Educación Financiera "Un sistema para vivir mejor". Innova Technology.

Roldan, P. (2019). Google. Obtenido de ECOMIPEDIA: https://economipedia.com/definiciones/finanzas-personales.html

WIKI EOI. (2013). El emprendedor. Capacidad de gestión y funciones en Proyectos de negocio. Obtenido de https://www.eoi.es/wiki/index.php/El_emprendedor._Capacidad_de_gesti\%C3\%B3n_y_f unciones_en_Proyectos_de_negocio

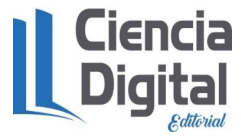




\section{PARA CITAR EL ARTÍCULO INDEXADO}

Cevallos Vique, V. O., Valverde Aguirre, P. E., Orna Hidalgo, L. A., \& Cargua Pilco, E. (2020). La educación financiera y su incidencia en la capacidad de gestión de los comerciantes de la empresa pública municipal Mercado de Productores Agrícolas San Pedro de Riobamba (EPEMMPA). Visionario Digital, 4(2), 5-21. https://doi.org/10.33262/visionariodigital.v4i2.1201

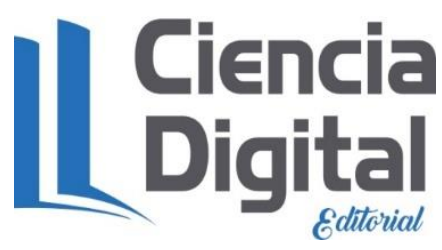

El artículo que se publica es de exclusiva responsabilidad de los autores y no necesariamente reflejan el pensamiento de la Revista Visionario Digital.

El artículo queda en propiedad de la revista y, por tanto, su publicación parcial y/o total en otro medio tiene que ser autorizado por el director de la Revista Visionario Digital.
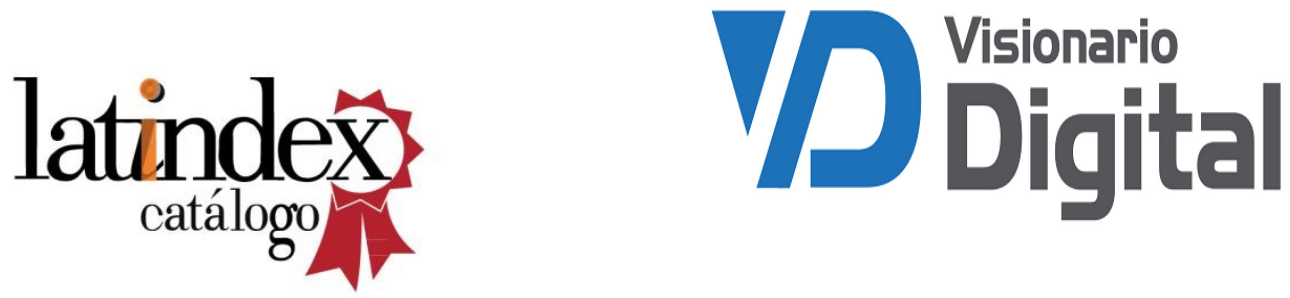